\title{
Properties of Slow Oscillation during Slow-Wave Sleep and Anesthesia in Cats
}

\author{
Sylvain Chauvette, ${ }^{1}$ Sylvain Crochet, ${ }^{1,2,3}$ Maxim Volgushev, ${ }^{4}$ and Igor Timofeev ${ }^{1}$ \\ ${ }^{1}$ Département de Psychiatrie et de Neurosciences, The Centre de Recherche Université Laval Robert-Giffard, Laval University, Québec, Québec, Canada \\ G1J 2G3, ${ }^{2}$ Inserm, Unité 1028, Centre National de la Recherche Scientifique, Unité Mixte de Recherche 5292, Lyon Neuroscience Research Center, Integrative \\ Physiology of Brain Arousal System Team, F-69000 Lyon, France, ${ }^{3}$ University Lyon 1, F-69000 Lyon, France, and ${ }^{4}$ University of Connecticut-Storrs, Department of \\ Psychology, Storrs, Connecticut 06269-1020
}

Deep anesthesia is commonly used as a model of slow-wave sleep (SWS). Ketamine-xylazine anesthesia reproduces the main features of sleep slow oscillation: slow, large-amplitude waves in field potential, which are generated by the alternation of hyperpolarized and depolarized states of cortical neurons. However, direct quantitative comparison of field potential and membrane potential fluctuations during natural sleep and anesthesia is lacking, so it remains unclear how well the properties of sleep slow oscillation are reproduced by the ketamine-xylazine anesthesia model. Here, we used field potential and intracellular recordings in different cortical areas in the cat to directly compare properties of slow oscillation during natural sleep and ketamine-xylazine anesthesia. During SWS cortical activity showed higher power in the slow/delta $(0.1-4 \mathrm{~Hz})$ and spindle $(8-14 \mathrm{~Hz})$ frequency range, whereas under anesthesia the power in the gamma band $(30-100 \mathrm{~Hz})$ was higher. During anesthesia, slow waves were more rhythmic and more synchronous across the cortex. Intracellular recordings revealed that silent states were longer and the amplitude of membrane potential around transition between active and silent states was bigger under anesthesia. Slow waves were mostly uniform across cortical areas under anesthesia, but in SWS, they were most pronounced in associative and visual areas but smaller and less regular in somatosensory and motor cortices. We conclude that, although the main features of the slow oscillation in sleep and anesthesia appear similar, multiple cellular and network features are differently expressed during natural SWS compared with ketamine-xylazine anesthesia.

\section{Introduction}

Slow-wave sleep (SWS) is characterized by large-amplitude slow waves in the electroencephalogram (Blake and Gerard, 1937). Their repetitive and more or less regular appearance during slowwave sleep and under anesthesia is commonly referred to as slow oscillation, which consists of an alternation of active (Up) and silent (Down) states in the cortico-thalamic network (Steriade et al., 1993a,b,c; Cowan and Wilson, 1994). During active states, neocortical neurons are depolarized, reveal vigorous synaptic activities, and may fire action potentials, whereas during silent states, they are hyperpolarized, display few synaptic events, and do not fire spikes (Contreras and Steriade, 1995; Steriade et al., 2001; Timofeev et al., 2001; Chauvette et al., 2010). Intracellular

Received May 9, 2011; revised July 26, 2011; accepted Aug. 26, 2011.

Author contributions: I.T. designed research; S.Ch., S.Cr., and I.T. performed research; S.Ch., M.V., and I.T. analyzed data; S.Ch., S.Cr., M.V., and I.T. wrote the paper.

This study was supported by Canadian Institutes of Health Research Grants MOP-37862 and MOP-67175, National Science and Engineering Research Council of Canada Grant 298475, and National Institute of Neurological Disorders and Stroke Grants 1R01-NS060870 and 1R01-NS059740 (I.T.). M.V. received startup funds from University of Connecticut. S.Ch. is a Canadian Institutes of Health Research fellow. S.Cr. is funded by Agence Nationale de la Recherche, France. I.T. is a Fonds de la Recherche en Santé du Québec Research Scholar. We are grateful to Sergiu Ftomov for excellent technical support, Josée Seigneur for logistic support, and Monty Escabi and Stanislav Volgushev for advice on data processing.

The authors declare no competing financial interests.

Correspondence should be addressed to Igor Timofeev, The Centre de Recherche Université Laval Robert-Giffard Local F-6500, 2601 de la Canardière, Québec, QC, Canada, G1J 2G3. E-mail: igor.timofeev@phs.ulaval.ca.

DOI:10.1523/JNEUROSCI.2339-11.2011

Copyright $\odot 2011$ the authors $\quad 0270-6474 / 11 / 3114998-11 \$ 15.00 / 0$ recordings from non-anesthetized cats (Steriade et al., 2001; Timofeev et al., 2001; Rudolph et al., 2007; Chauvette et al., 2010) and rats (Mahon et al., 2006; Okun et al., 2010) demonstrated that, when local field potential (LFP) displays slow waves, cortical and striatal neurons alternate between active and silent states. In contrast, when LFP displays activated patterns, normally associated with wakefulness and rapid eye movement (REM) sleep, the membrane potential of cortical neurons does not express silent states and no large-amplitude LFP waves occur (Matsumura, 1979; Steriade et al., 2001; Timofeev et al., 2001; Rudolph et al., 2007; Okun et al., 2010; Constantinople and Bruno, 2011). Similar activities were found during active but not quiet wakefulness in mice (Crochet and Petersen, 2006; Poulet and Petersen, 2008; Gentet et al., 2010). After prolonged periods of wakefulness, local slow waves can occur in otherwise awake animals (Vyazovskiy et al., 2011).

Experiments on cats and rats anesthetized with ketaminexylazine or urethane revealed a slow cortical activity similar to that observed during natural SWS (Steriade et al., 1993a,b,c; Contreras and Steriade, 1995; Haider et al., 2006; Volgushev et al., 2006; Haider et al., 2007; Hasenstaub et al., 2007; Luczak et al., 2007; Chauvette et al., 2010; Sharma et al., 2010). Anesthesiainduced slow oscillation appears more regular than during SWS (Steriade et al., 1993b; Wolansky et al., 2006; Clement et al., 2008), and its frequency was higher with ketamine-xylazine than urethane anesthesia (Steriade et al., 1993b; Sharma et al., 2010), indicating that properties of the slow oscillation are not fixed but 
depend on experimental conditions. Recordings from different cortical areas show a preferential origin of slow waves at specific locations (Massimini et al., 2004; Volgushev et al., 2006; Kurth et al., 2010; Nir et al., 2011), suggesting regional specificity.

Ketamine-xylazine anesthesia is extensively used as a model of sleep slow oscillation; however, no direct comparison of the properties of slow oscillation during anesthesia and SWS had been done so far. To address this issue, we performed LFP and intracellular recordings across different cortical areas in both naturally sleeping and ketamine-xylazine-anesthetized cats. We found that, despite apparent similarities, major differences exist between SWS and anesthesia.

\section{Materials and Methods}

Experiments were performed in accordance with the guideline of the Canadian Council on Animal Care and approved by the Laval University Committee on Ethics and Animal Research. The cats were purchased from an established animal breeding supplier. Good health conditions of all animals were certified by the supplier and determined on arrival to animal house by physical examination, which was performed by animal facilities technicians and a veterinarian in accordance with requirements of the Canadian Council on Animal Care. The animals were used for experiments in 5-20 d from arrival to the local animal house.

\section{General description and database}

We recorded field potentials, extracellular unit, and intracellular activities of cortical neurons in various cortical areas of cats during natural sleep or in cats anesthetized with ketamine-xylazine. We used recordings from database acquired over the past 10 years for neurons recorded from suprasylvian gyrus (associative cortex, 36 cats in acute experiments and four cats in chronic experiments), postcruciate gyrus (somatosensory cortex, five cats in acute experiments and one cat in chronic experiments), precruciate gyrus (motor cortex, one cat in acute experiments and one cat in chronic experiments), and posterior marginal gyrus (primary visual cortex, two cats in chronic experiments). To complement our database with multisite LFP recordings and to collect additional intracellular data, we performed additional experiments in motor (one acute and one chronic), somatosensory (four chronic), and visual (three acute and one chronic) cortices. Because in the same experiment we were able to record from several cortical areas, altogether we used 43 cats for acute experiments and eight cats for chronic experiments.

\section{Preparation}

Experiments on anesthetized animals. For acute experiments, the cats of both sexes were anesthetized with ketamine and xylazine (10-15 and 2-3 $\mathrm{mg} / \mathrm{kg}$, i.m., respectively). All pressure points and tissues to be incised were infiltrated with lidocaine $(0.5 \%)$. The animals were paralyzed with gallamine triethiodide $(20 \mathrm{mg} / \mathrm{kg})$ and artificially ventilated, maintaining the end-tidal $\mathrm{CO}_{2}$ concentration at 3.5-3.8\%. A permanent sleep-like state, as ascertained by continuous recording of the LFP, was maintained throughout the experiments by administering additional doses of ketamine and xylazine (5 and $1 \mathrm{mg} / \mathrm{kg}$, respectively) or just ketamine (5 $\mathrm{mg} / \mathrm{kg}$ ). The additional anesthesia was administrated when LFP slow waves were absent for $20-30 \mathrm{~s}$. The body temperature was monitored by a rectal probe and maintained at $37^{\circ} \mathrm{C}$ via a feedback-controlled heating blanket. The heart rate was continuously monitored ( $90-110$ beats $/ \mathrm{min})$. The stability of intracellular recordings was reinforced by cisternal drainage, bilateral pneumothorax, and filling the hole made for recordings with a solution of $4 \%$ agar.

Experiments on non-anesthetized animals. Chronic experiments were conducted using an approach similar to that described previously (Steriade et al., 2001; Timofeev et al., 2001). For implantation of recording chamber and electrodes, four cats (data from the old database) were anesthetized with sodium pentobarbital (30 mg/ $\mathrm{kg})$, and four cats were anesthetized with isoflurane (0.75-2\%), in accordance with the current recommendation of the Committee for Animal Care of Laval University. All other procedures were very similar. Briefly, before surgery, the animal was given a dose of pre-anesthetic, which was composed of ketamine (15 $\mathrm{mg} / \mathrm{kg})$, buprenorphine $(0.01 \mathrm{mg} / \mathrm{kg})$, and acepromazine $(0.3 \mathrm{mg} / \mathrm{kg})$. After site shaving and cat intubation for gaseous anesthesia, the site of incision was washed with at least three alternating passages of proviodine solution and alcohol. Lidocaine $(0.5 \%)$ was injected at the site of incision and applied at all pressure points. During surgery, electrodes for LFP recordings, electromyogram (EMG) from neck muscle, and electrooculogram (EOG) were implanted and fixed with acrylic dental cement. Custom-made recording chambers were fixed for future intracellular recordings. Eight to 10 screws were fixed to the cranium. Four bolts were covered in the dental cement that also covered bone-fixed screws, permanently implanted electrodes, and fixed recording chamber to allow future head-restrained recordings without any pressure point. Throughout the surgery, the body temperature was maintained at $37^{\circ} \mathrm{C}$ using a water-circulating thermo-regulated blanket. Hearth beat and oxygen saturation were continuously monitored using a pulse oximeter (Rad-8; MatVet), and the level of anesthesia was adjusted to maintain a heart beat at $110-120$ per minute. A lactate Ringer's solution $\left(10 \mathrm{ml} \cdot \mathrm{kg}^{-1} \cdot \mathrm{h}^{-1}\right.$, i.v.) was given during the surgery. After the surgery, cats were given buprenorphine $(0.01 \mathrm{mg} / \mathrm{kg})$ or anafen $(2 \mathrm{mg} / \mathrm{kg})$ twice a day for $3 \mathrm{~d}$ and baytril $(5 \mathrm{mg} / \mathrm{kg}$ ) once a day for $7 \mathrm{~d}$. Approximately 1 week was allowed to animals to recover from the surgery before the first recording session. Usually, 2-3 d of training were sufficient for cats to remain in headrestrained position for $2-4 \mathrm{~h}$ and display one to three full sleep-wake cycles, including periods of quiet wakefulness, SWS, and REM sleep. The recordings were performed up to $40 \mathrm{~d}$ after the surgery.

\section{Recordings}

All recordings were done in a Faraday chamber. Using AM 3000 amplifiers (A-M Systems) with custom modifications, LFPs were recorded in marginal gyrus [visual cortex (areas 17 and 18)], suprasylvian gyrus [associative cortex (areas 5, 7, and 21)], ectosylvian gyrus [auditory cortex (areas 22 and 50)], postcruciate gyrus [somatosensory cortex (area 3)], precruciate gyrus [motor cortex (areas 4 and 6)], and frontal gyrus [medial prefrontal cortex (area 32)] using tungsten electrodes ( $2 \mathrm{M} \Omega$; bandpass filter, $0.1 \mathrm{~Hz}$ to $10 \mathrm{kHz}$ ). We aimed to implant electrodes at $1 \mathrm{~mm}$ below the cortical surface. Intracellular recordings were performed using glass micropipettes filled with $2.5 \mathrm{M}$ potassium acetate and having a resistance of 30-70 M $\Omega$. A high-impedance amplifier with active bridge circuitry (Neurodata IR-283 amplifiers; Cygnus Technology; low-pass filter, $10 \mathrm{kHz}$ ) was used to record the membrane potential and to inject current into the neurons. Intracellular recordings were performed from areas $3,4,7$, and 17 according to an atlas (Reinoso-Suarez, 1961). In acute experiments, a chloridized silver wire, placed between the skull and the temporal muscle, was used as a reference electrode. In chronic experiments, a silver wire was fixed either in the frontal bone over the sinus cavity or over the cerebellum. All electrical signals were sampled at 20 $\mathrm{kHz}$ and digitally stored on Vision (Nicolet). At the end of experiments, the cats were anesthetized with ketamine-xylazine to record LFP activities with the same electrodes as during natural states of vigilance. After collecting $1 \mathrm{~h}$ or more of recordings, the cats were killed with a lethal dose of pentobarbital (50 $\mathrm{mg} / \mathrm{kg}$, i.v.).

\section{Data analysis}

Electrographic recordings were analyzed offline using custom-written routines in IgorPro (WaveMetrics) and MATLAB (MathWorks) environment. To measure the amplitude of slow oscillation, we used intracellular recordings. The amplitude of field potential recordings depends on the activity of cellular elements in the region but also on the electrode location relative to electrical dipole(s). Because the cortical thickness is different in different areas, the amplitude of LFP at $1 \mathrm{~mm}$ from the surface of the pia can give erroneous estimation of slow oscillation amplitude. It is generally assumed that, during slow-wave activity, the membrane potential of neurons has a bimodal distribution (Metherate and Ashe, 1993), and this was the case in the majority of our recordings (Fig. 1a1-b1,a3-b3). However, when transitions between active and silent states were slow and their duration was comparable with the time a neuron spent in those states, the membrane potential distribution was not bimodal, although silent states could be clearly identified (Fig. 1a2$b 2, a 4-b 4)$. To resolve this problem, we analyzed periods around transi- 


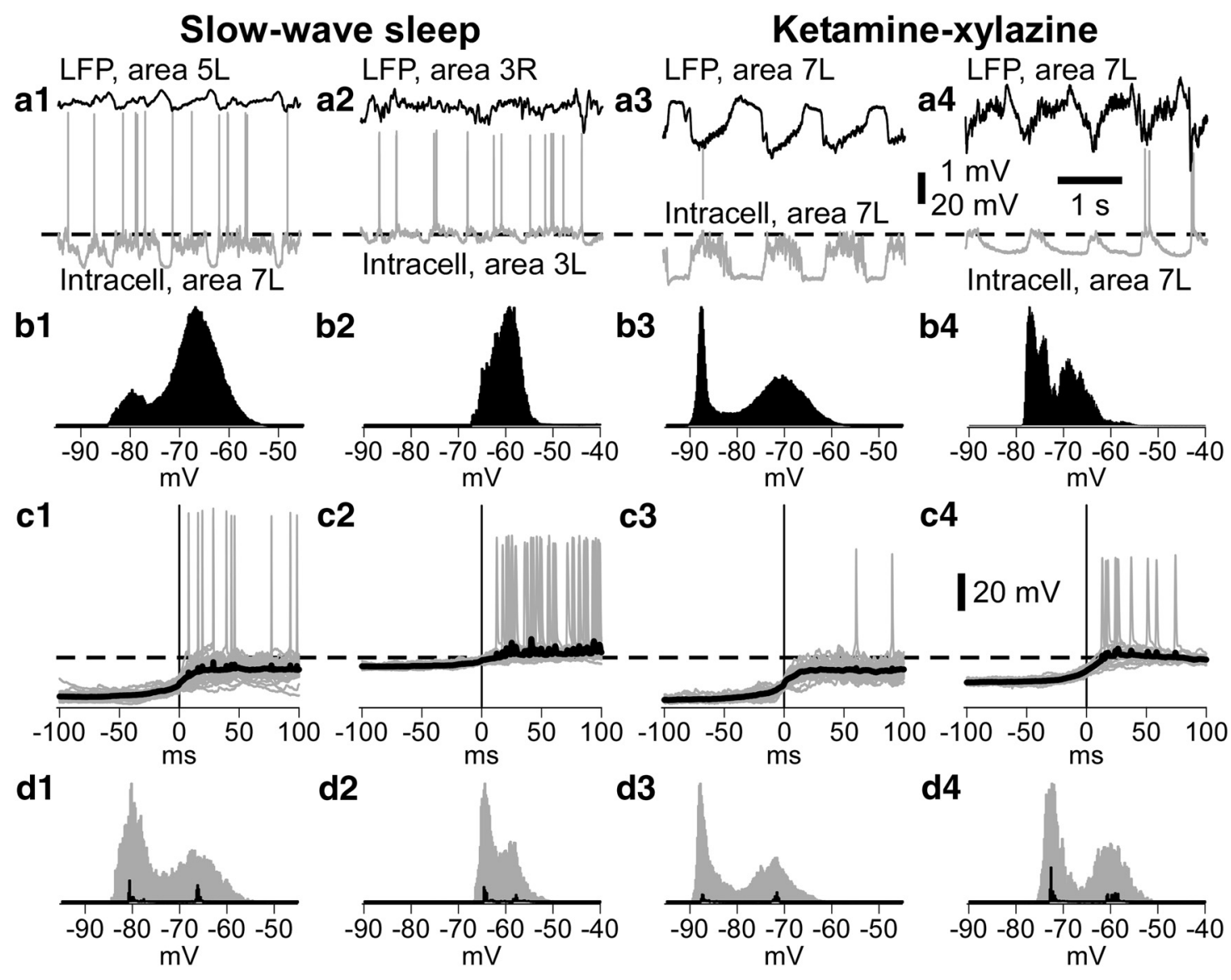

Figure 1. Calculation of the amplitude of slow oscillation in the membrane potential during transitions from silent to active states. Columns 1 and 3 show recordings during slow-wave sleep and ketamine-xylazine anesthesia with clearly bimodal distribution of membrane potential, whereas columns 2 and 4 show recordings with clear silent and active states but no clear bimodality of the membrane potential distribution. $\boldsymbol{b} \mathbf{1 - b 4}$, Histograms of membrane potential distribution from $60 \mathrm{~s}$ segments from cells shown in $\boldsymbol{a} \mathbf{1}-\boldsymbol{a} \mathbf{4}$. $\boldsymbol{c} \mathbf{1}-\boldsymbol{c} \mathbf{4}$, Transitions from silent to active states (time 0 corresponding to the time at half-amplitude) and event-triggered average (thick black traces). All transitions of a 60 s segment are displayed in gray. d1-d4, Histograms of all gray segments shown in c (gray histogram). Histograms of event-triggered average are shown in black. Note the bimodal distribution for all recordings with this method.

tions from silent to active states. We first selected time at half-amplitude of transitions from silent to active state from 1 min segment of a recording. We then selected segments of $\pm 100 \mathrm{~ms}$ from that point (Fig. 1c) and compiled a histogram using all these segments (Fig. 1d, gray histograms). On all occasions, the distribution of the membrane potential was bimodal; however, because of the natural fluctuation of the membrane potential, the two maxima were broad. Therefore, from selected segments, we computed averages (Fig. 1c, black traces) and calculated the histogram of membrane potential distribution from these averaged traces (Fig. 1d, black histogram). The results gave clear bimodal distribution of membrane potential (Fig. 1). This method allowed unbiased analysis of all recorded neurons.

The frequency composition of electric signals was calculated using fast Fourier transformation of multiple $20 \mathrm{~s}$ segments of LFP traces. From power spectrum of each data segment, we calculated the integral power in the frequency ranges between 0.1 and $4 \mathrm{~Hz}$ for slow and delta activities, 8 and $14 \mathrm{~Hz}$ for spindles, and 30 and $100 \mathrm{~Hz}$ for gamma-band fluctuations. We also calculated autocorrelation of field potential and membrane potential recordings and coherence between pairs of simultaneously recorded LFPs. Coherence was calculated using the MATLAB (R2011b) function mscohere, which uses Welch's averaged modified periodogram method to calculate the magnitude squared coherence estimate. Periodic Hamming windows with $50 \%$ overlap were used to obtain eight equallength sections of each signal. Coherence function was calculated with a step of $0.1526 \mathrm{~Hz}$. Then, coherence in three frequency ranges [slow and delta $(0.2-4 \mathrm{~Hz})$, spindle $(8-14 \mathrm{~Hz})$, and gamma $(30-100 \mathrm{~Hz})]$ was calculated by averaging the values within the respective range. Unless specified, all numerical values are given as mean $\pm \mathrm{SD}$. Specific statistical tests are indicated throughout.

\section{Results}

We used two different strategies to compare properties of LFP, extracellular unit activity, and intracellular membrane potential traces during natural SWS and ketamine-xylazine anesthesia. Field potentials were recorded with chronically implanted electrodes during natural sleep-wake cycle and thereafter under ketamine-xylazine anesthesia. This allowed us to compare LFP signals pairwise, with each pair consisting of recordings made from the same electrode in the same animal during natural sleep and anesthesia. Intracellular recordings from neurons in different cortical areas were made sequentially during natural sleep or anesthesia; therefore, intracellular data were analyzed as groups.

On one occasion (Fig. 2), we were able to record LFP and extracellular unit activities of the same neuron in the somatosensory cortex (postcruciate gyrus, area 3) during waking state, SWS, and ketamine-xylazine anesthesia (Fig. $2 b$, insets). During waking state characterized by high-frequency low-amplitude activity in the LFP, the neuronal firing was continuous and did not reveal periodic patterns (Fig. 2). Only central peak, but no additional peaks, were present in the autocorrelogram of the firing of the neurons (Fig. 2b, left). During transition to SWS, evidenced by characteristic largeamplitude slow waves in the LFP and EEG, the neuronal firing became grouped and occurred during depth-negative phases of the LFP (Fig. 2a). Several minutes after injection of ketamine-xylazine anesthesia, the rhythmicity and the frequency of slow waves increased, and the firing rate increased and became highly rhythmic 
a

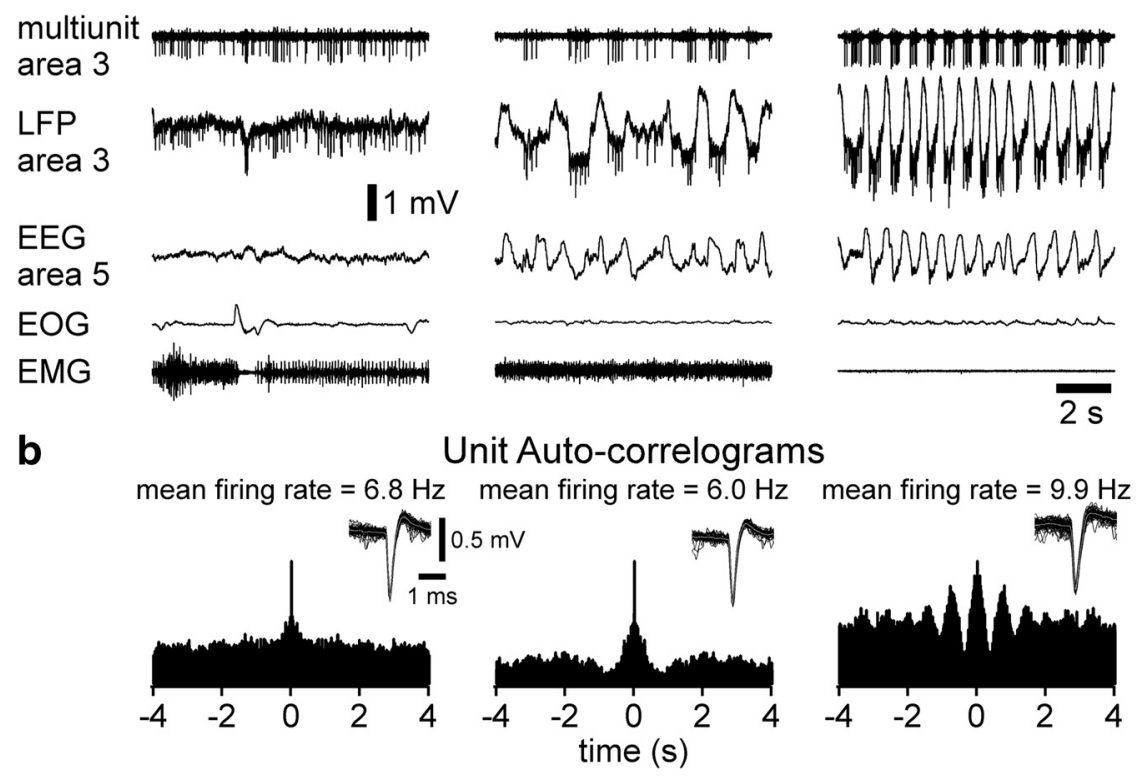

Figure 2. Fragments of continuous electrographic recordings during waking, slow-wave sleep, and ketamine-xylazine anesthesia. $\boldsymbol{a}$, Traces of multiunit activity and local field potential in cortical area 3, EEG from area 5, EOG, and EMG recorded in one cat during indicated conditions. Corresponding recordings were obtained with the same electrodes. $\boldsymbol{b}$, Autocorrelograms of the unit recording from the neuron shown in $\boldsymbol{a}$. Insets, Fifty spikes and their average (gray line) of the unit shown in $\boldsymbol{a}$ for the three recorded states. Note a dramatic increase in rhythmicity of cortical activities under ketamine-xylazine anesthesia.

(Fig. 2). Similar to SWS, the neuronal firing occurred during depthnegative phases of the LFP (Fig. 2a). The rhythmicity of neuronal firing increased, as evidenced by multiple alternating peaks and troughs in the autocorrelogram (Fig. $2 b$, right).

To examine whether these changes in activity could be generalized to other cortical areas, we have studied cortical electrographic activities in cat neocortex during natural SWS and during ketaminexylazine anesthesia in precruciate gyrus, postcruciate gyrus, frontal gyrus, marginal gyrus, suprasylvian gyrus, and ectosylvian gyrus (Fig. 3a). Figure $3 b$ shows results of a typical experiment in which field potentials were recorded simultaneously from multiple cortical locations using chronically implanted electrodes, first during an episode of natural SWS, and then during ketamine-xylazine anesthesia. Slow oscillation is clearly present in each individual trace in this example: all recordings in Figure $3 b$ express slow, large-amplitude waves and fulfill a formal criterion of a low $(<4 \mathrm{~Hz})$ over high $(>4$ $\mathrm{Hz}$ ) frequencies power ratio $>3$ (Mukovski et al., 2007). An averaged ratio for all 11 locations was $6.11 \pm 5.8$ during anesthesia and $8.18 \pm 6.9$ (mean \pm SEM) during SWS. However, the whole picture of the slow oscillation recorded simultaneously from multiple locations was clearly different during SWS compared with anesthesia. During SWS, slow waves were always more prominent in suprasylvian gyrus (Fig. $3 b$, locations $8-10$ ). In other cortical areas, slow waves were of smaller amplitude and even not evident in some cycles (Fig. $3 b$, left). In all cortical regions, the intervals between slow waves were highly irregular. After the injection of ketamine-xylazine anesthesia, the pattern of slow oscillation changed (Fig. $3 b$, right). The slow waves became more regular and rhythm like. They also were more generalized across different cortical regions, so that every wave was evident in all simultaneously recorded channels.

The differences between patterns of slow waves in field potential observed during SWS and anesthesia were corroborated by results of intracellular recordings. Figure $3 c-f$ shows examples of membrane potential traces of neurons recorded in different areas of the neocortex during SWS or anesthesia. All neurons recorded during SWS or under ketamine-xylazine anesthesia exhibited alternating active (depolarized) and silent (hyperpolarized) states. However, during anesthesia, the slow oscillation appears to be more rhythmic. Furthermore, during anesthesia, the slow oscillation was of overall higher amplitude, and the silent states were of longer duration than in SWS (Fig. 3c-f).

To validate and quantify these differences, we have compared, for SWS and anesthesia, and for different cortical regions, the following parameters: spectral composition, rhythmicity and coherence of the slow oscillation, its amplitude, and the duration of silent states.

Less power in the slow and spindle range but more in the high-frequency range during ketamine-xylazine anesthesia than in natural sleep Although LFPs recorded in a same animal in both SWS and ketamine-xylazine anesthesia expressed clear slow activities, spectral analysis revealed substantial differences in their frequency composition. Figure 4 shows an example of LFP recorded in one experiment in somatosensory cortex (area 3 ) of the right hemisphere during an SWS episode and then under ketamine-xylazine anesthesia. A higher rhythmicity of slow oscillation and an increase in fast activities (see filtered traces in Fig. $4 a$ ) can be clearly seen in the recording obtained under anesthesia. Power spectra of $20 \mathrm{~s} \mathrm{epochs}$ around the illustrated segments of recordings revealed marked differences in three characteristic frequency ranges (Fig. 4b). Power in the slow and delta frequency range $(0.1-4 \mathrm{~Hz})$ and at spindle frequencies $(8-14 \mathrm{~Hz})$ in suprasylvian gyrus was significantly higher during SWS (Fig. 4c). A similar pattern was found in different regions, but the difference did not reach significance level. When data from all investigated areas were pooled together, the power was significantly decreased for slow/delta $(p=0.003$, two-tailed onesample $t$ test) and for spindle frequency $(p<0.0001)$ ranges, but the gamma range activities were dramatically increased under anesthesia (Fig. $4 c, p<0.0001$ ).

\section{Stronger rhythmicity of neuronal activity during anesthesia than in natural sleep}

LFP, extracellular unit, and membrane potential of cortical neurons showed a stronger rhythmicity of slow oscillation under anesthesia (Figs. 2, 3). To quantify the rhythmicity, we computed autocorrelations of $20 \mathrm{~s}$ segments of LFP and intracellular recordings (40 neurons) during SWS and under anesthesia (Fig. 5). During SWS, the slow waves appeared with only weak, if at all, rhythmicity. This is evidenced by the low amplitude or the absence of secondary peaks with a period of 1-2 s in the autocorrelograms (Fig. 5, left column). Under anesthesia, autocorrelograms of both LFP and intracellular recordings from all four investigated areas expressed several clear secondary peaks. These recurrent peaks occurring at regular intervals are indicative of a strong rhythmicity of the slow oscillation (Fig. $5 a, b$, right columns). The amplitude of the secondary peak, usually occurring between 0.5 and $1.5 \mathrm{~s}$, was significantly higher during anesthesia than in SWS for all studied areas (Fig. 5c), indicating a strong 
increase in the rhythmicity of the slow oscillation under ketamine-xylazine anesthesia.

Higher coherence of slow oscillation between different regions during ketamine-xylazine anesthesia than during SWS

Simultaneous LFP recordings made from multiple cortical regions during SWS and subsequent anesthesia (Fig. $3 b$ ) suggest that slow waves occur more synchronously in different cortical regions during anesthesia than in SWS. To assess this relation quantitatively, we have calculated pairwise coherence of the LFPs recorded at different locations during SWS and compared it with the coherence of LFPs recorded during anesthesia from the same pairs of chronically implanted electrodes (Fig. 6). The coherence was calculated for three frequency ranges (Fig. 6): slow and delta $(0.2-4 \mathrm{~Hz})$, spindle $(8-14 \mathrm{~Hz})$, and gamma (30-100 $\mathrm{Hz}$ ). The coherence between different cortical regions during anesthesia was high for all investigated ranges of frequencies (Fig. $6 c$, green-yellow-brown plots). It was systematically higher during the anesthesia than during natural SWS for signal components in the slow and delta range $(0.2-4 \mathrm{~Hz}$, $0.594 \pm 0.102$ vs $0.507 \pm 0.149, p<0.001$, $n=45$ pairs of recordings from the same electrodes during anesthesia and SWS, Wilcoxon's paired test) and in the gamma frequency range $(30-100 \mathrm{~Hz}, 0.43 \pm 0.031$ vs $0.399 \pm 0.024, p<0.003)$. In this example, for the spindle range, the difference did not reach significance level $(0.435 \pm 0.094$ vs $0.425 \pm 0.031, p=0.147)$. In the scatter plots in which coherence during anesthesia is plotted against coherence during the SWS for each pair of recorded electrodes, most points are located above the diagonal for slow/delta and gamma frequencies (Fig. $6 a, b)$. Higher coherence during anesthesia is particularly evident in coherence difference (anesthesia minus SWS) color (red-whiteblue) plots by a strong domination of blue colors, indicating a higher coherence during anesthesia (Fig. $6 c$ ). To validate these results, we performed the same analysis on $n=165$ pairs (four cats) of SWS and anesthesia recordings. In each pair of electrodes, the coherence was calculated in epochs of $15 \mathrm{~s}$ (10-12 epochs for each recording) and then averaged for that pair of electrodes. For the whole sample, the coherence in all three frequency ranges was significantly higher during the anesthesia than during SWS: $0.524 \pm 0.109$ versus $0.487 \pm 0.127, p<0.001$, Wilcoxon's paired test, for slow and delta range $(0.2-4 \mathrm{~Hz}) ; 0.442 \pm 0.1$ versus $0.429 \pm 0.088, p=0.008$ for the spindle range $(8-14 \mathrm{~Hz})$; and $0.4 \pm 0.051$ versus $0.386 \pm 0.029, p<0.001$ for the gamma range $(30-100 \mathrm{~Hz})$. Thus, slow waves as well as activity in the spindle

C

d

e

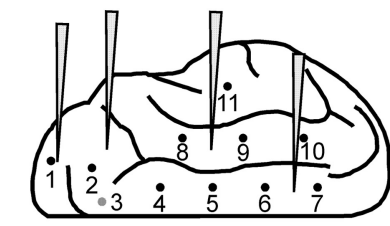

1. Precruciate Gyrus

2. Postcruciate Gyrus

3. Frontal Gyrus

4-7. Marginal Gyrus

8-10. Suprasylvian Gyrus

11. Ectosylvian Gyrus

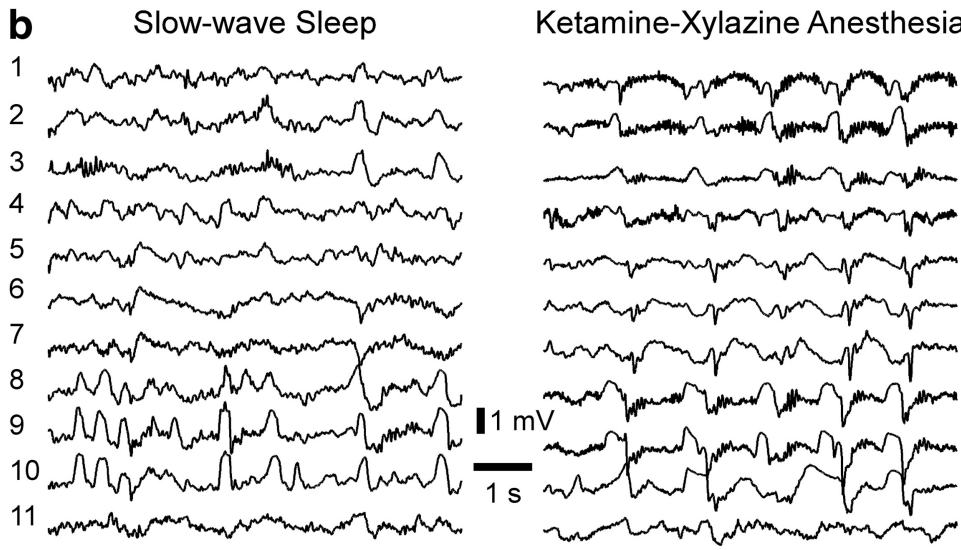

Precruciate Gyrus

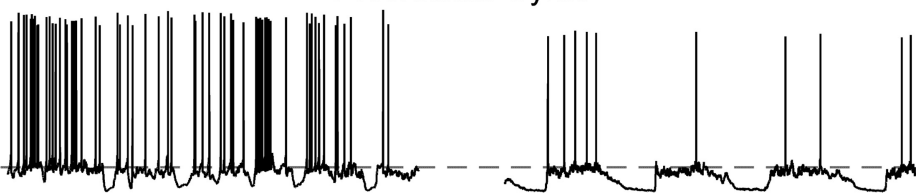

Postcruciate Gyrus

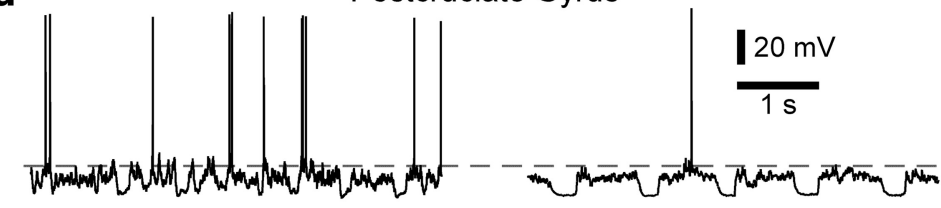

Marginal Gyrus

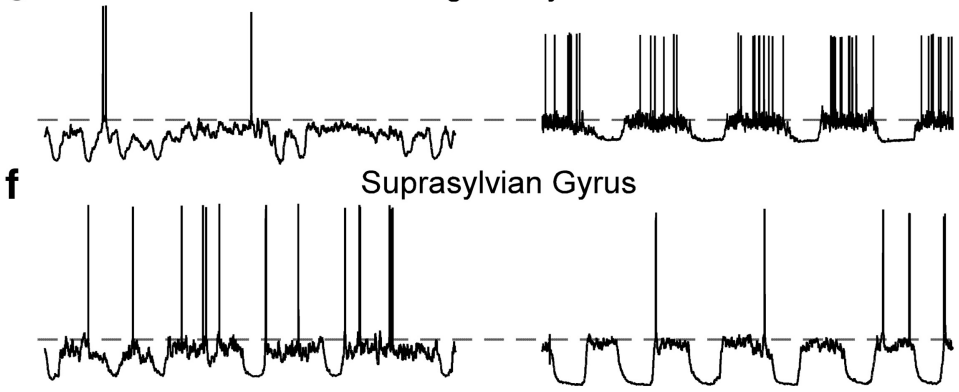

Figure 3. Typical field potential and intracellular recordings from different cortical areas during natural slow-wave sleep and ketamine-xylazine anesthesia. $\boldsymbol{a}$, Schematic representation of the location of electrodes for local field potential (black dots with numbers indicate recording sites for traces in $\boldsymbol{b}$ ) and for intracellular recordings (pipette drawing, for traces in $\mathbf{c}-\boldsymbol{f}$ ). $\boldsymbol{b}$, Field potentials recorded simultaneously in different cortical areas in one cat using the same set of chronically implanted electrodes, either during slow-wave sleep (left column) or under ketamine-xylazine anesthesia (right column). In slow-wave sleep recordings, note the most prominent slow oscillation in suprasylvian gyrus (electrode locations $8-10$ ). In anesthesia recordings, note the high synchrony of slow waves at all locations. c-f, Typical segments of intracellular recordings during slow-wave sleep (left column) or ketamine-xylazine anesthesia (right column). Intracellular recordings: $\boldsymbol{c}$, precruciate gyrus; $\boldsymbol{d}$, postcruciate gyrus; $\boldsymbol{e}$, marginal gyrus; and $\boldsymbol{f}$, suprasylvian gyrus. The scale is the same for all intracellular traces. Gray dotted lines indicate $-60 \mathrm{mV}$.

and gamma frequency ranges were more synchronized during anesthesia compared with natural SWS.

Silent states are more prominent during anesthesia than in SWS

Intracellular recordings suggested that, for a given cortical area, the slow oscillation was usually of smaller amplitude and silent states of a shorter duration during SWS compared with anesthe- 


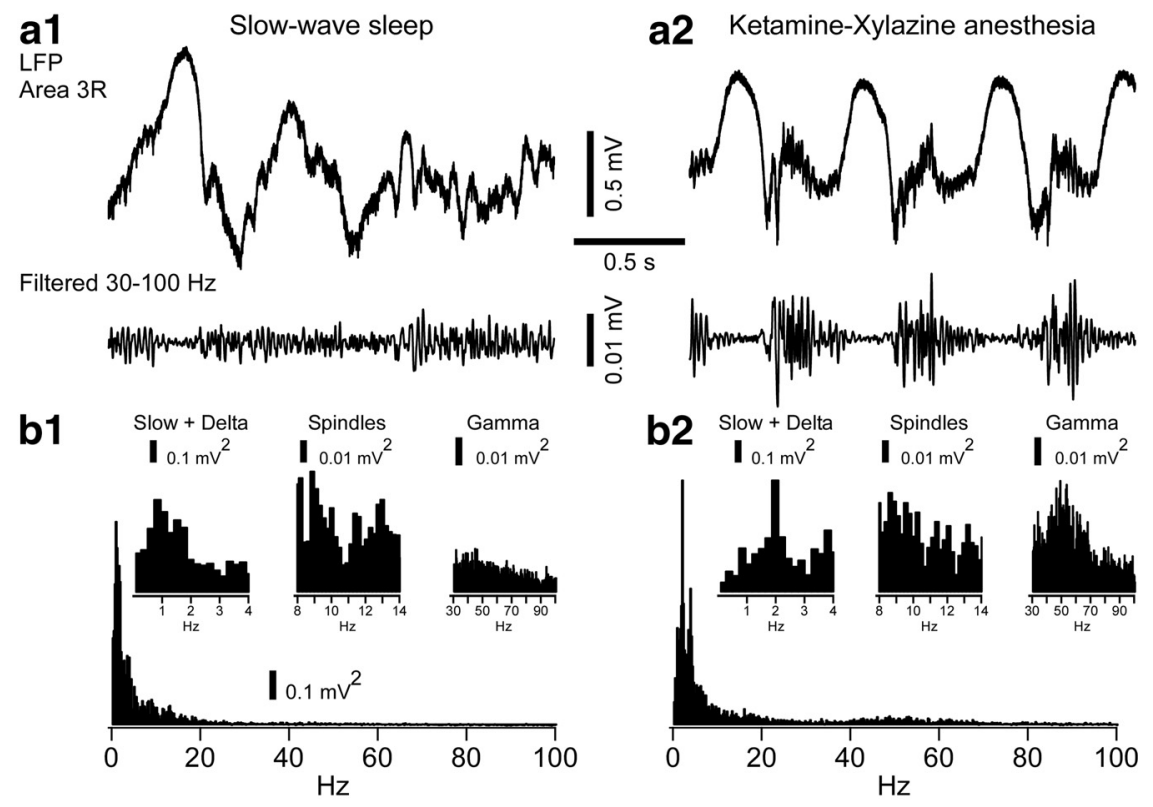

C

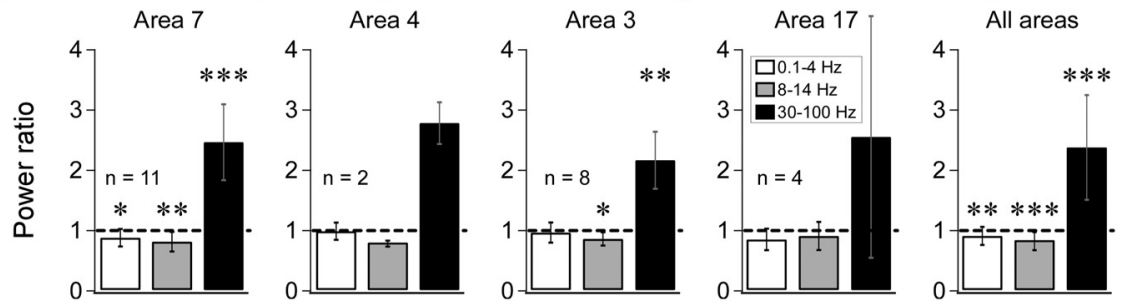

Figure 4. Spectral composition of local field potentials is different during slow-wave sleep and anesthesia. $\boldsymbol{a}$, Segment of LFP recorded in the right somatosensory cortex (area 3, top trace) and its high-frequency component ( $30-100 \mathrm{~Hz}$, bottom trace) during SWS ( $\boldsymbol{a} \mathbf{1})$ and under ketamine-xylazine anesthesia ( $\boldsymbol{a} \mathbf{2}$ ). Recordings in $\boldsymbol{a} \mathbf{1}$ and $\boldsymbol{a} \mathbf{2}$ are from the same chronically implanted electrode in the same animal. $\boldsymbol{b} \mathbf{1}, \boldsymbol{b} \mathbf{2}$, Power spectra $(0-100 \mathrm{~Hz})$ of a 20 s epoch of the same recording as in $\boldsymbol{a} \mathbf{1}, \boldsymbol{a} 2$. Insets show a zoom-in of slow and delta $(0.1-4 \mathrm{~Hz})$, spindle $(8-14 \mathrm{~Hz})$, and gamma $(30-100 \mathrm{~Hz})$ frequency ranges. $c$, Mean ratio (ketaminexylazine anesthesia/SWS) of the integral power in the three frequency ranges $(0.1-4,8-14$, and $30-100 \mathrm{~Hz})$. The mean power ratio was calculated from 10 pairs of 20 s epochs of LFP recorded from the same electrodes during anesthesia and SWS in different cortical locations. Note the higher power during SWS in $0.1-4$ and $8-14 \mathrm{~Hz}$ but the large increase in gamma power under ketamine-xylazine anesthesia. ${ }^{*} p<0.05,{ }^{* *} p<0.01,{ }^{* * *} p<0.001$, Wilcoxon's signed rank test for area specific comparisons, one-sample $t$ test for pooled data.

sia (examples in Fig. $3 c-f$ ). To assess these differences quantitatively, we measured the duration of silent states at half-amplitude of transition to and from silent states. Comparison of silent states in all recordings performed under anesthesia to all recordings made during SWS revealed that the duration of silent states was significantly shorter during SWS in each investigated area and for all areas pooled together (Fig. 7a1). The proportion of time spent in silent state, calculated as the ratio of time a cell spent in silent state to the total time analyzed, was also higher under anesthesia than in SWS. This difference was significant both when recordings from all cortical regions were pooled together and when data were segregated by cortical area (Fig. $7 b 1$ ).

The amplitude of slow oscillation recorded intracellularly was calculated as described in Materials and Methods (Fig. 1). For the whole sample, when recordings made from different cortical areas were pooled together, the amplitude of slow oscillation was significantly larger under ketamine-xylazine anesthesia than in natural SWS (Fig. 7c1). When the comparison was made separately for recordings obtained in each area, the amplitude of silent states during anesthesia was significantly higher than in SWS in area 3 but not in other areas (Fig. $7 c 1$ ).
Next, we compared the duration and amplitude of silent states in membrane potential of neurons recorded during slow oscillation between different areas in the same experimental condition of either SWS or anesthesia. This comparison revealed area-specific differences in silentstate duration in both SWS and anesthesia (Fig. 7a2,b2). A striking difference between SWS and anesthesia was observed for the amplitude of slow oscillation, which showed area-specific differences during SWS that were not present under anesthesia (Fig. 7c2).

\section{Discussion}

In this study, we demonstrated that, despite a generally similar appearance of slow oscillation during deep SWS and anesthesia, their quantitative comparison revealed multiple features that are distinct in these two brain states. (1) During anesthesia, the rhythmicity of slow oscillation in the LFP and intracellular recordings was higher than in SWS. (2) During anesthesia, the LFP power in slow and delta $(0.1-4 \mathrm{~Hz})$ and in spindle $(8-14 \mathrm{~Hz})$ frequency ranges was decreased, but the power of gamma activity was doubled. (3) During anesthesia, the coherence of LFP in different areas and for all investigated frequency ranges was higher than during SWS. (4) Intracellular recordings revealed areaspecific differences of slow oscillation amplitude during SWS with larger amplitudes in visual and associative areas and smaller amplitudes in somatosensory area. Anesthesia increased the amplitude of slow oscillation in most areas and abolished differences between areas. (5) The duration of silent states was also area-specific, both during SWS and under anesthesia; however, in every area, anesthesia increased the duration of silent states to $148-240 \%$ compared with its value during SWS.

\section{Effects of anesthesia on cortical neurons}

Slow oscillation is composed of two different phases: an active phase when cortical neurons are depolarized and may fire action potentials, and a silent phase when cortical neurons are hyperpolarized and silent (Steriade et al., 1993b). This general pattern of slow oscillation was seen in both SWS and ketamine-xylazine anesthesia. Silent state is a period of disfacilitation (absence of synaptic activity) during which the membrane potential of cortical neurons is primarily dominated by potassium leak conductance (Wilson et al., 1983; Metherate and Ashe, 1993; Contreras et al., 1996; Timofeev et al., 1996, 2001; Wilson and Kawaguchi, 1996). Active states are associated with high levels of excitatory and inhibitory conductances of both synaptic and intrinsic origin (Wilson and Kawaguchi, 1996). Excitation and inhibition can be approximately balanced (Haider et al., 2006) or dominated by in- 


\section{Slow-wave sleep Ketamine-Xylazine}

a Autocorrelations

a Local field potential

Intracellular Local field potential Intracellular
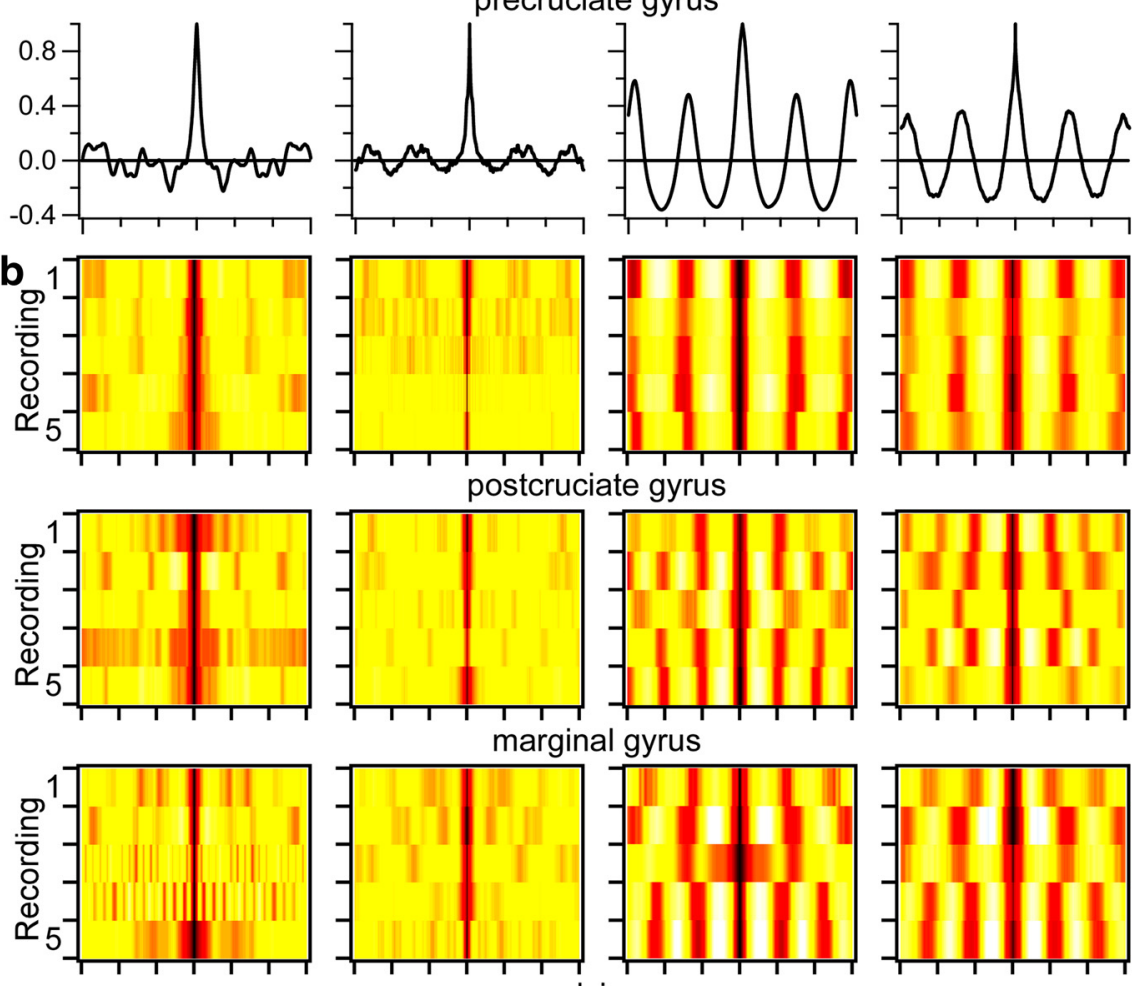

suprasylvian gyrus
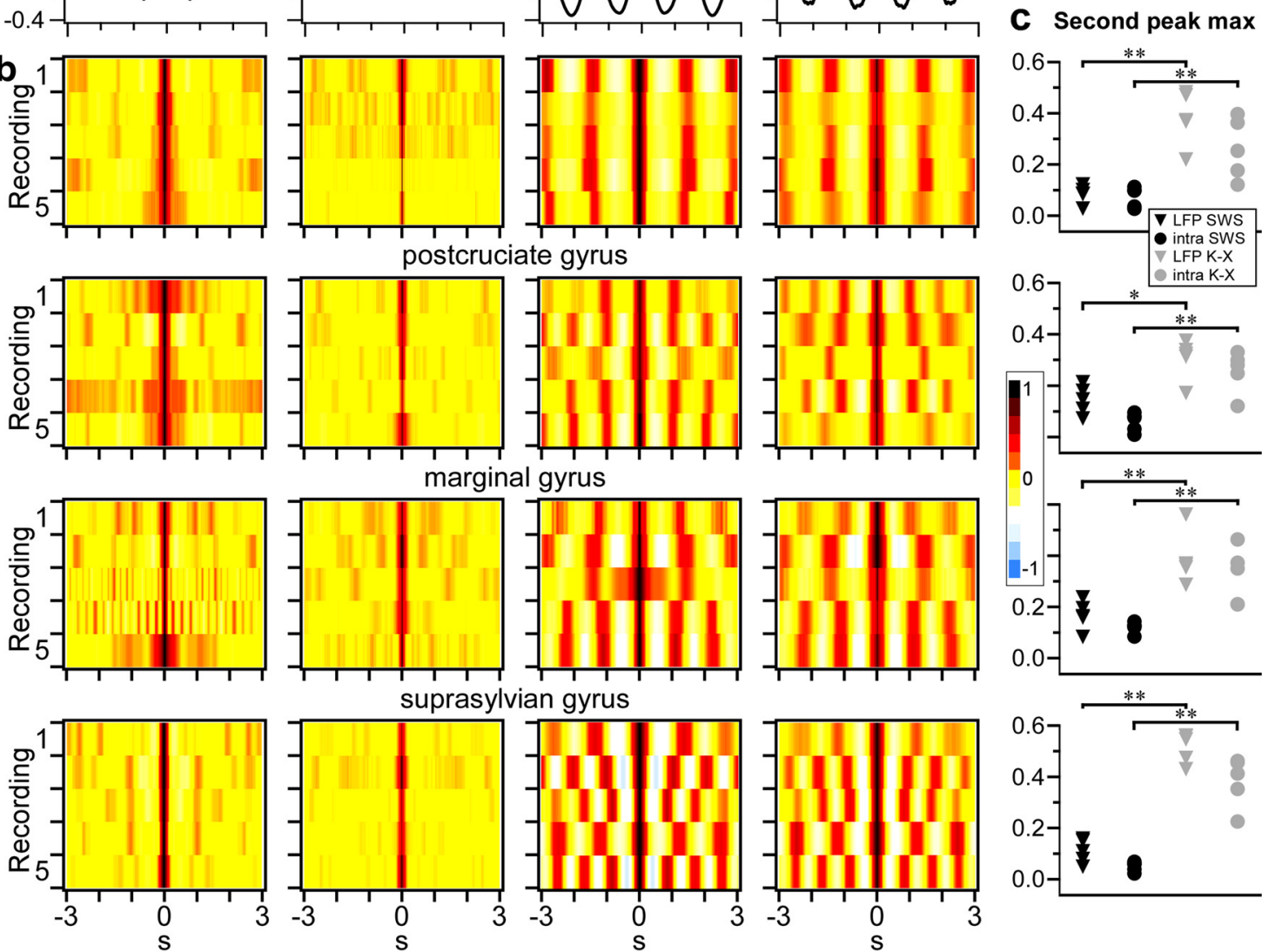

Figure 5. Rhythmicity of slow waves in local field potential and membrane potential is higher during ketamine-xylazine anesthesia than in SWS. $\boldsymbol{a}, \boldsymbol{b}$, Autocorrelograms of local field potential (columns 1 and 3) or membrane potential (columns 2 and 4) recorded either during slow-wave sleep (left columns) or during ketamine-xylazine anesthesia (right columns) in the precruciate gyrus. Examples in $\boldsymbol{a}$ and recording 1 of precruciate gyrus in color-coded panels in $\boldsymbol{b}$ show the same data. $\boldsymbol{b}$, Each panel shows five typical examples of color-coded autocorrelations in four different cortical areas as indicated. Note the strong rhythmicity of both field potential and intracellular activities under ketamine-xylazine anesthesia as evidenced by periodically alternating peaks (red) and troughs (yellow) in the autocorrelograms. c, The magnitude of the second peak in autocorrelation of LFP and membrane potential during SWS and anesthesia in the four cortical areas. In all cases, the magnitude of the second peak was significantly higher under anesthesia (two-tailed Mann-Whitney test, ${ }^{*} p<0.05,{ }^{* *} p<0.01$ ).

hibition (Rudolph et al., 2007) depending on experimental condition. Ketamine is a noncompetitive, use- and voltagedependent antagonist of NMDA receptors, leading to a partial blockade of glutamatergic neurotransmission (MacDonald et al., 1991; Liu et al., 2006). Therefore, the use of ketamine should decrease excitatory conductance. Indeed, in all investigated cortical regions, we observed under ketamine-xylazine anesthesia an increase of both the duration of silent states and the proportion of time spent in silent states (Fig. $7 a, b)$. This implies a decrease in the duration of active states. This observation is congruent to a recent finding demonstrating that a hypofunction of NMDA receptor attributable to genetic modulation or antagonist application increases the length of silent states (Fellin et al., 2009). A recent study demonstrated that a decrease in inhibition had similar effects: it shortened active states and prolonged silent states (Sanchez-Vives et al., 2010). This suggests that a fine tuning of excitation and inhibition in each cortical area is responsible for the generation of a partic- ular frequency of slow oscillation and that manipulations leading to a decrease of the efficiency of either excitation or inhibition will shorten active states and lengthen silent states. Several additional effects of ketamine on cortical neurons had been reported. Ketamine application hyperpolarizes cortical neurons via a block of HCN1 channels, enhances synaptic responses (Chen et al., 2009), and enhances $\mathrm{Ca}^{2+}$ spikes and dendritic excitability in pyramidal cells in vitro, but not in vivo because synaptic activity reduces dendritic spiking in cortical neurons (Potez and Larkum, 2008). Ketamine also inhibits nicotinic cholinoreceptors (Rudolph and Antkowiak, 2004); however, because the firing of cholinergic neurons is strongly reduced during both SWS and anesthesia (Steriade and McCarley, 2005; Boucetta and Jones, 2009), it is unlikely that ketamine induces slow oscillations via this mechanism. Ketamine may also potentiate GABA responses (Rudolph and Antkowiak, 2004; Alkire et al., 2008), but, at anesthetic doses, it acts exclusively on $\alpha 6 \beta 2 \delta$ and $\alpha 6 \beta 3 \delta$ receptors (Hevers et al., 


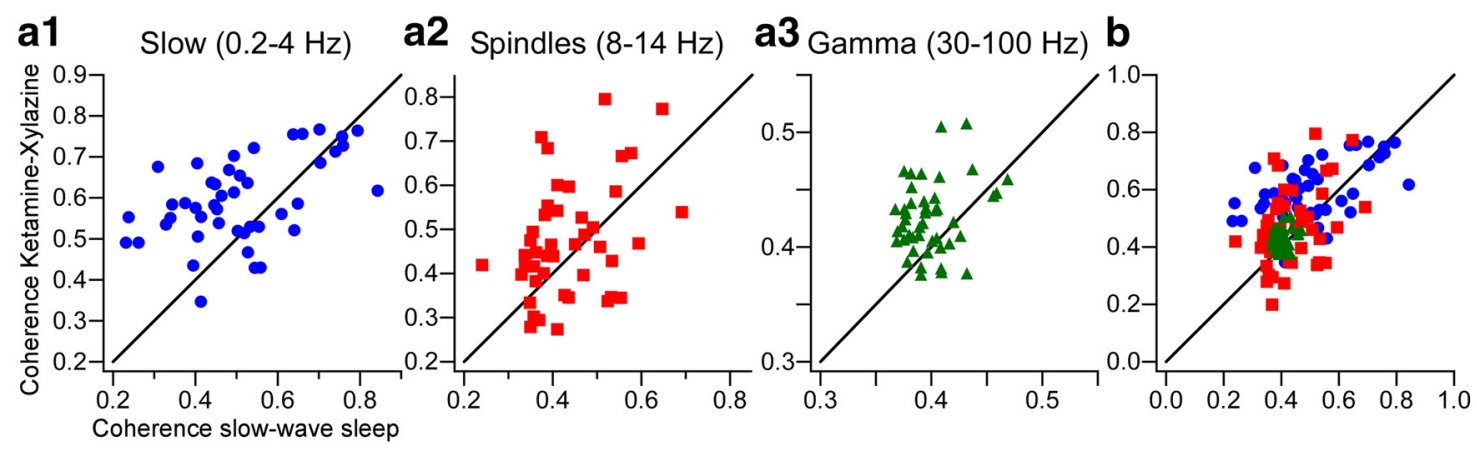

coherence value ketamine xylazine I coherence difference (ketamine-xylazine - slow-wave sleep)

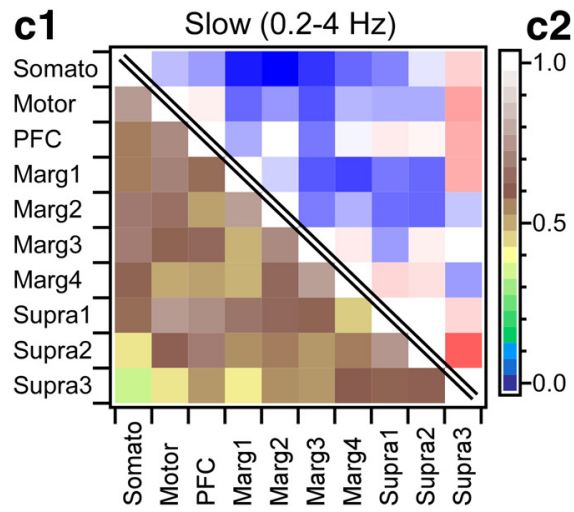

c2

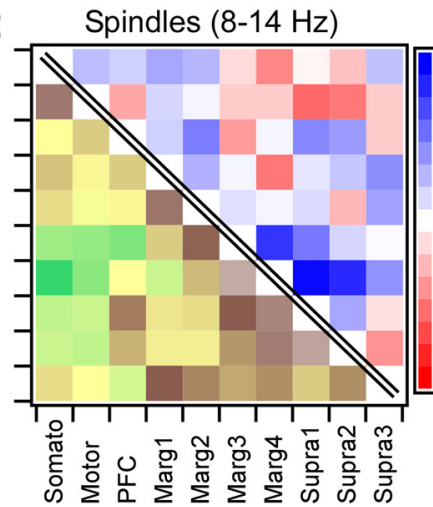

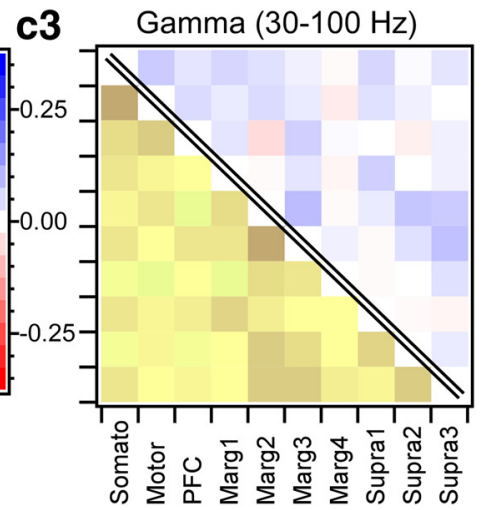

Figure 6. Higher coherence of slow oscillation during ketamine-xylazine anesthesia than in slow-wave sleep. Coherence in the slow/delta frequency range (0.2- 4 Hz; $\boldsymbol{a} 1)$, in the spindle frequency range $(8-14 \mathrm{~Hz} ; \boldsymbol{a} 2)$, and in the gamma frequency range $(30-100 \mathrm{~Hz} ; \boldsymbol{a} 3)$ of LFPs recorded during ketamine-xylazine anesthesia plotted against coherence calculated from recordings made with the same electrodes but during slow-wave sleep (LFP recordings shown in Fig. $3 b$ ). $\boldsymbol{b}$, Data from $\boldsymbol{a}$ 1-a $\mathbf{3}$ but plotted on the same scale. $\boldsymbol{c}$, (panels above the diagonal, red-white- blue color code). The difference (anesthesia - slow-wave sleep) in coherence for each pair of recording sites for slow/delta frequencies ( $\mathbf{c}$ ), spindle frequencies ( $\mathbf{2}$ ), and gamma frequencies ( $\mathbf{c} 3$ ). Blue colors indicate higher coherence during anesthesia; red colors indicate higher coherence during sleep. Note that most pairs show a stronger coherence during ketamine-xylazine anesthesia than during sleep (seen as a positive difference, blue). c (panels below the diagonal, green-yellow-brown), Coherence values for each pair of recording sites under ketamine-xylazine anesthesia. Recording sites are indicated as follows (see Fig. 3a). Somato, postcruciate gyrus; Motor, precruciate gyrus; PFC, frontal gyrus; Marg, marginal gyrus; Supra, suprasylvian gyrus. 1 is the most anterior, and 4 is the most posterior site.

2008), which, in mammalian brain, were found only in the granular layer of cerebellum (Pirker et al., 2000). GABAergic action of ketamine unlikely contributes to the generation of active and silent states in the thalamocortical system.

We observed a significant increase in the amplitude of intracellularly recorded slow oscillation (Fig. 7c) that was likely attributable to a more pronounced hyperpolarization during silent states induced by ketamine-xylazine anesthesia. In all recorded areas, we observed a more pronounced hyperpolarization during silent states in anesthetized cats compared with SWS. However, in chronic experiments, the reference electrode remained implanted for weeks or months and thus could develop some direct current polarization. Therefore, we were not confident in comparing absolute values of the membrane potential, but we compared the difference between active and silent states (Fig. 7). Xylazine is an agonist for $\alpha 2$ adrenergic receptors, which are abundant in the neocortex (Hedler et al., 1981; Nicholas et al., 1993). The $\alpha 2$ adrenoreceptor agonist clonidine activates a potassium current in rat spinal cord neurons (Sonohata et al., 2004). Noradrenaline also increases potassium conductance in mouse entorhinal cortex (Pralong and Magistretti, 1995). In the locus ceruleus, noradrenaline and $\alpha 2$ adrenoreceptor agonists activate potassium current, whereas $\alpha 2$ antagonist blocks them (Arima et al., 1998). Activation of $\alpha 2$ adrenoreceptor increases cortical activity via closure of h-channels (Wang et al., 2007). Therefore, the action of xylazine could contribute to both the longer duration and the bigger difference in amplitude between active and silent states observed in our experiments in anesthetized animals.

\section{Anesthesia, sleep, consciousness}

Higher brain functions occurring during conscious states require coordinated spatiotemporal firing in large neuronal constellations with hierarchal organization (Konorski, 1967; Buzsáki, 2010) involving internally and externally generated signals (Edelman, 2003). A minimal time of 0.5-1 s is required to be conscious (Libet et al., 1967; Edelman, 2003). Continuous neuronal firing of cortical, thalamic, and striatal neurons occurs during persistent active (depolarized) network states in awake animals (Steriade et al., 2001; Timofeev et al., 2001; Mahon et al., 2006; Vyazovskiy et al., 2009) as well as during REM sleep (Hirsch et al., 1983; Steriade et al., 2001; Timofeev et al., 2001). This fulfills the requirement for uninterrupted coordinated activity; therefore, some forms of consciousness could occur during both waking state and REM sleep. On the contrary, during SWS, cortical, thalamocortical, and striatal neurons oscillate between active and silent states (Hirsch et al., 1983; Steriade et al., 2001; Timofeev et al., 2001; Mahon et al., 2006). Active states are depolarized states in cortical and striatal neurons, but in thalamocortical neurons, they are associated with multiple IPSPs originating from burst firing of reticular thalamic neurons (Contreras and Steriade, 1995; Timofeev and Steriade, 1996). During SWS, dominated by slow oscillation with a period of $\sim 1 \mathrm{~s}$, the duration of silent 


\section{Silent state duration}
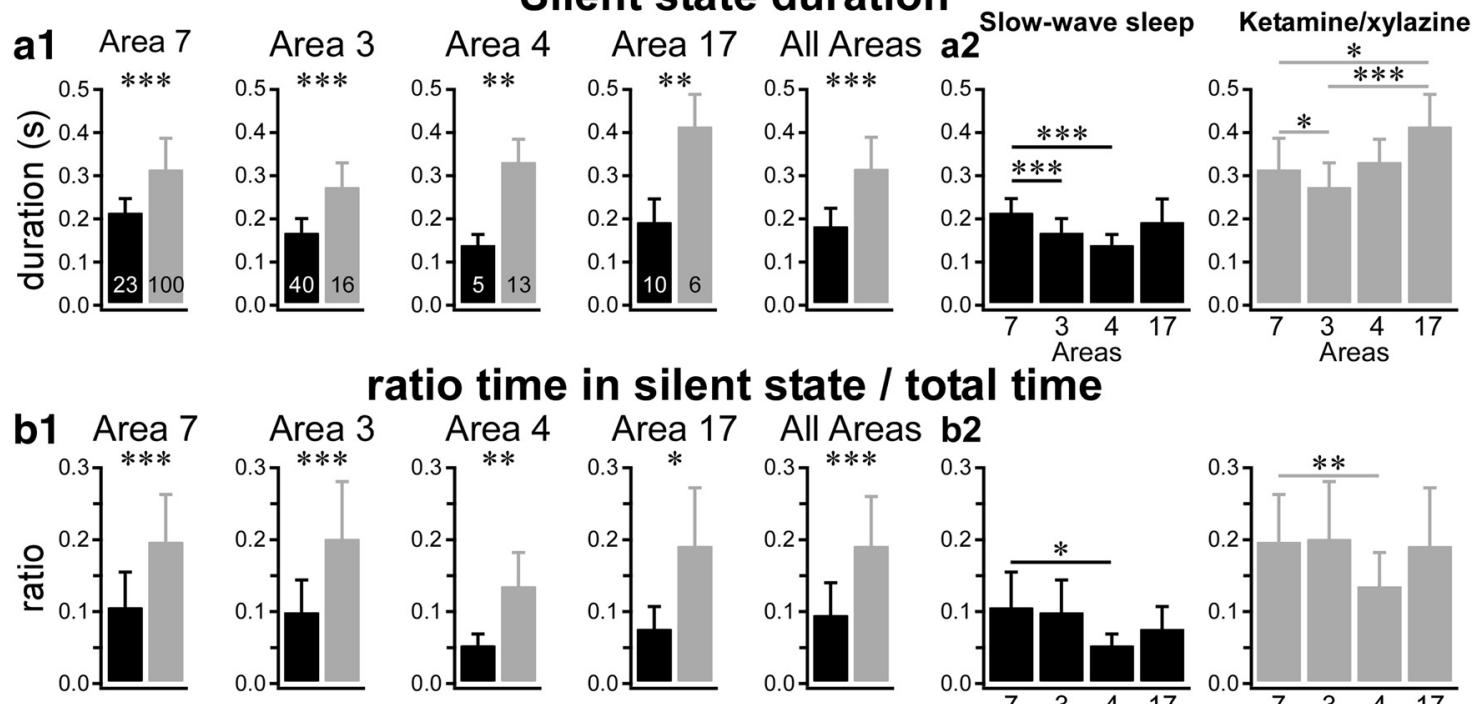

3
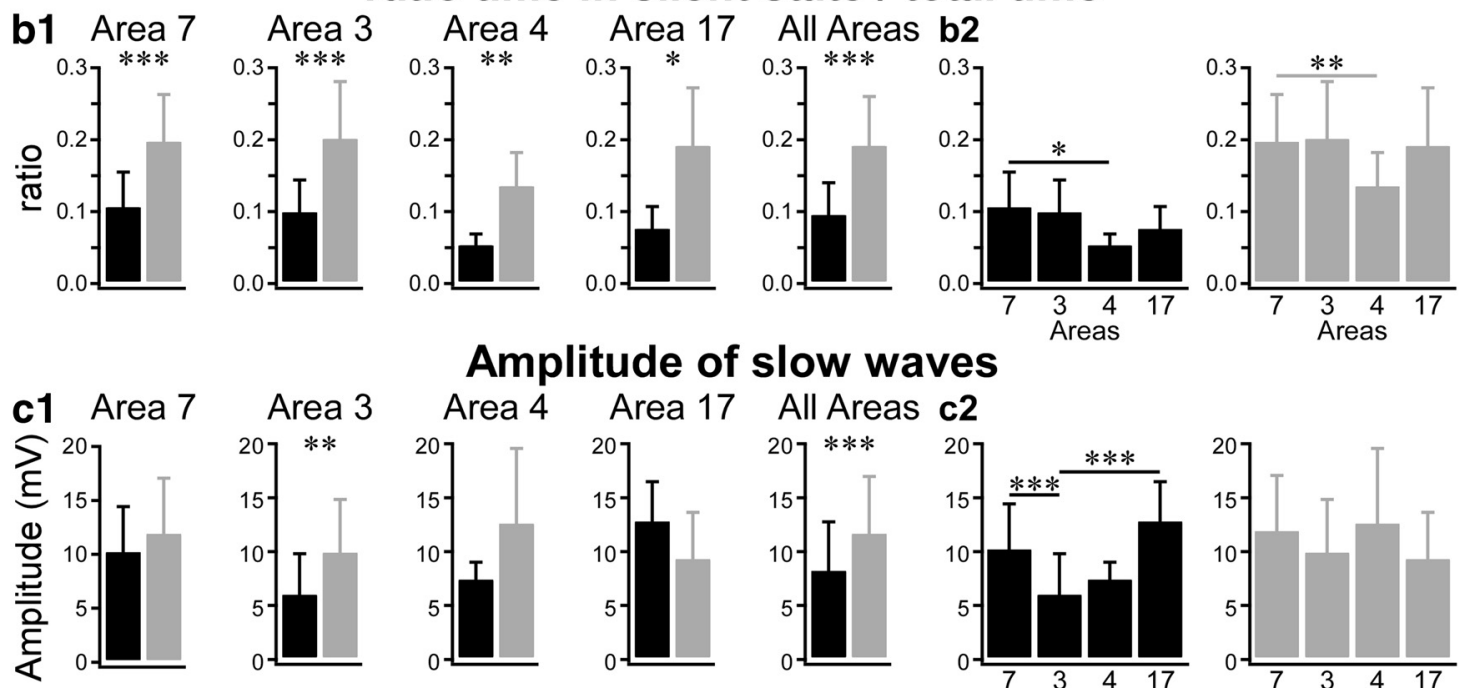

Amplitude of slow waves
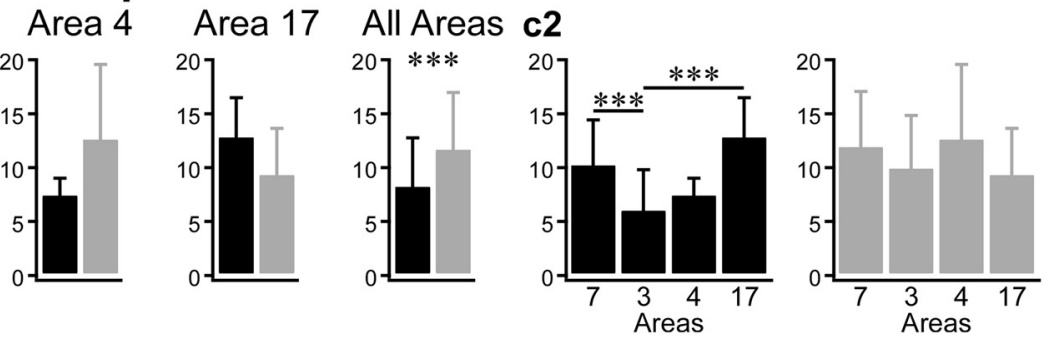

Figure 7. Silent states are more prominent during anesthesia than in SWS. $\boldsymbol{a} 1, \boldsymbol{a} 2$, Mean duration of silent states measured at half-amplitude transitions from active to silent and from silent to active states in intracellular recordings during sleep (black) or anesthesia (gray). $\boldsymbol{a 1}$, Comparison of the mean silent state duration during SWS and under anesthesia for each cortical area and pooled data from all areas. $\boldsymbol{a} 2$, Comparison between different areas during SWS and anesthesia. Note that duration of silent states is significantly longer under anesthesia than during SWS. $\boldsymbol{b} \mathbf{1}, \boldsymbol{b 2}$, Mean ratio of time spent in silent state over total time. Comparison scheme as in $\boldsymbol{a}$. Note the longer time spent in silent states during anesthesia than during SWS. $\mathbf{c 1 ,} \mathbf{c 2}$, Mean amplitude of membrane potential shift between silent and active state during slow oscillation measured from intracellular recordings. Comparison scheme as in $\boldsymbol{a}$. Note that pooled data show significantly larger membrane potential transition amplitude under anesthesia compared with SWS. Note that amplitude of membrane potential transition between silent and active states is significantly different between cortical areas during SWS but not during anesthesia. The number of cells recorded in each condition is indicated within each bar in $\mathbf{a 1} .{ }^{*} p<0.05,{ }^{* *} p<0.01,{ }^{* * *} p<0.001 . a 1-\mathrm{c1}$, Mann-Whitney test for $n<15$, unpaired $t$ test with Welch's correction for larger samples. $\boldsymbol{a} 2-\mathbf{c 2}$, Kruskal-Wallis test with Dunn's correction.

states is $\sim 150-200 \mathrm{~ms}$, and neurons spend $5-10 \%$ of time in silent states (Fig. 7). Therefore, in deep SWS, the continuous neuronal firing lasts $<1 \mathrm{~s}$, thus impairing the emergence of conscious experience. Slow-wave activity is highest and more global at the onset of sleep period and then progressively decreases toward the end of sleep (Achermann and Borbély, 2003; Vyazovskiy et al., 2009; Nir et al., 2011). It is well accepted that the expression of slow oscillation in cortical areas is correlated with activities in those areas during wake (Huber et al., 2004). Like in humans, most of sensory information in cat arrives via the visual system. Therefore, we expected, and it was indeed the case, to obtain larger amplitude of slow oscillation in area 17 (Fig. 7). The neurons in area 7 also showed larger amplitude of slow oscillation, because this is a polysensory area with visual dominance (Bignall, 1967). It is likely that, in rodent cortex, the biggest amplitude of slow waves would be recorded in somatosensory areas, because this is the leading sensory system in these animals. During slow waves, neurons in large cortical territories reveal almost simultaneous alternations of activity and silence (Volgushev et al., 2006). Because of the decreased slow-wave activity toward the end of the sleep period, the duration of active states, estimated from extracellular unit recordings, increases and often lasts longer than 1 s (Vyazovskiy et al., 2009). Therefore, some form of conscious processes are possible during late SWS, and the majority of dreams reported outside REM episodes was found during these late periods of sleep, when cortical neurons display longer uninterrupted firing stretches (Payne and Nadel, 2004).

Recent reviews discuss whether general anesthesia-induced amnesia, rather than loss of consciousness, plays a more important role in the overall effects of anesthesia (Rudolph and Antkowiak, 2004; Alkire et al., 2008; Tononi and Koch, 2008). All agreed that deep SWS might be considered as an unconscious state. When ketamine-xylazine anesthesia is used, the duration of silent states approximately doubles, and the cortical network spends almost $20 \%$ of time in silent states (Fig. 7 ), preventing an essential condition of conscious states, i.e., persistent neuronal firing. The synchrony of slow waves under ketamine-xylazine anesthesia is higher than during SWS (Figs. $3,6)$. Therefore, the active states occur more synchronously across cortical areas, and virtually all cortical neurons become silent simultaneously, making logical processing of information and conscious states during ketamine-xylazine anesthesia impossible. Overall, we suggest that the enhanced presence of 
slow waves under anesthesia compared with SWS may be used as a safe criterion to estimate annihilation of conscious state.

\section{References}

Achermann P, Borbély AA (2003) Mathematical models of sleep regulation. Front Biosci 8:s683-s693.

Alkire MT, Hudetz AG, Tononi G (2008) Consciousness and anesthesia. Science 322:876-880.

Arima J, Kubo C, Ishibashi H, Akaike N (1998) Alpha2-adrenoceptormediated potassium currents in acutely dissociated rat locus coeruleus neurones. J Physiol 508:57-66.

Bignall KE (1967) Comparison of optic afferents to primary visual and polysensory areas of cat neocortex. Exp Neurol 17:327-343.

Blake H, Gerard RW (1937) Brain potentials during sleep. Am J Physiol 119:692-703.

Boucetta S, Jones BE (2009) Activity profiles of cholinergic and intermingled gabaergic and putative glutamatergic neurons in the pontomesencephalic tegmentum of urethane-anesthetized rats. J Neurosci 29: $4664-4674$.

Buzsáki G (2010) Neural syntax: cell assemblies, synapsembles, and readers. Neuron 68:362-385.

Chauvette S, Volgushev M, Timofeev I (2010) Origin of active states in local neocortical networks during slow sleep oscillation. Cereb Cortex 20:2660-2674.

Chen X, Shu S, Bayliss DA (2009) Hcnl channel subunits are a molecular substrate for hypnotic actions of ketamine. J Neurosci 29:600-609.

Clement EA, Richard A, Thwaites M, Ailon J, Peters S, Dickson CT (2008) Cyclic and sleep-like spontaneous alternations of brain state under urethane anaesthesia. PLoS One 3:e2004.

Constantinople CM, Bruno RM (2011) Effects and mechanisms of wakefulness on local cortical networks. Neuron 69:1061-1068.

Contreras D, Steriade M (1995) Cellular basis of eeg slow rhythms: a study of dynamic corticothalamic relationships. J Neurosci 15:604-622.

Contreras D, Timofeev I, Steriade M (1996) Mechanisms of long-lasting hyperpolarizations underlying slow sleep oscillations in cat corticothalamic networks. J Physiol 494:251-264.

Cowan RL, Wilson CJ (1994) Spontaneous firing pattern and axonal projections of single corticostriatal neurons in the rat medial agranular cortex. J Neurophysiol 71:17-32.

Crochet S, Petersen CC (2006) Correlating whisker behavior with membrane potential in barrel cortex of awake mice. Nat Neurosci 9:608-610.

Edelman GM (2003) Naturalizing consciousness: a theoretical framework. Proc Natl Acad Sci U S A 100:5520-5524.

Fellin T, Halassa MM, Terunuma M, Succol F, Takano H, Frank M, Moss SJ, Haydon PG (2009) Endogenous nonneuronal modulators of synaptic transmission control cortical slow oscillations in vivo. Proc Natl Acad Sci U S A 106:15037-15042.

Gentet LJ, Avermann M, Matyas F, Staiger JF, Petersen CC (2010) Membrane potential dynamics of gabaergic neurons in the barrel cortex of behaving mice. Neuron 65:422-435.

Haider B, Duque A, Hasenstaub AR, McCormick DA (2006) Neocortical network activity in vivo is generated through a dynamic balance of excitation and inhibition. J Neurosci 26:4535-4545.

Haider B, Duque A, Hasenstaub AR, Yu Y, McCormick DA (2007) Enhancement of visual responsiveness by spontaneous local network activity in vivo. J Neurophysiol 97:4186-4202.

Hasenstaub A, Sachdev RN, McCormick DA (2007) State changes rapidly modulate cortical neuronal responsiveness. J Neurosci 27:9607-9622.

Hedler L, Stamm G, Weitzell R, Starke K (1981) Functional characterization of central alpha-adrenoceptors by yohimbine diastereomers. Eur J Pharmacol 70:43-52.

Hevers W, Hadley SH, Lüddens H, Amin J (2008) Ketamine, but not phencyclidine, selectively modulates cerebellar $\mathrm{GABA}_{\mathrm{A}}$ receptors containing $\alpha 6$ and $\delta$ subunits. J Neurosci 28:5383-5393.

Hirsch JC, Fourment A, Marc ME (1983) Sleep-related variations of membrane potential in the lateral geniculate body relay neurons of the cat. Brain Res 259:308-312.

Huber R, Ghilardi MF, Massimini M, Tononi G (2004) Local sleep and learning. Nature 430:78-81.

Konorski J (1967) Integrative activity of the brain: an interdisciplinary approach. Chicago: The University of Chicago.

Kurth S, Ringli M, Geiger A, LeBourgeois M, Jenni OG, Huber R (2010)
Mapping of cortical activity in the first two decades of life: a high-density sleep electroencephalogram study. J Neurosci 30:13211-13219.

Libet B, Alberts WW, Wright EW Jr, Feinstein B (1967) Responses of human somatosensory cortex to stimuli below threshold for conscious sensation. Science 158:1597-1600.

Liu J, Ji XQ, Zhu XZ (2006) Comparison of psychic emergence reactions after $(+/-)$-ketamine and (+)-ketamine in mice. Life Sci 78:1839-1844.

Luczak A, Barthó P, Marguet SL, Buzsáki G, Harris KD (2007) Sequential structure of neocortical spontaneous activity in vivo. Proc Natl Acad Sci U S A 104:347-352.

MacDonald JF, Bartlett MC, Mody I, Pahapill P, Reynolds JN, Salter MW, Schneiderman JH, Pennefather PS (1991) Actions of ketamine, phencyclidine and MK-801 on NMDA receptor currents in cultured mouse hippocampal neurones. J Physiol 432:483-508.

Mahon S, Vautrelle N, Pezard L, Slaght SJ, Deniau JM, Chouvet G, Charpier S (2006) Distinct patterns of striatal medium spiny neuron activity during the natural sleep-wake cycle. J Neurosci 26:12587-12595.

Massimini M, Huber R, Ferrarelli F, Hill S, Tononi G (2004) The sleep slow oscillation as a traveling wave. J Neurosci 24:6862-6870.

Matsumura M (1979) Intracellular synaptic potentials of primate motor cortex neurons during voluntary movement. Brain Res 163:33-48.

Metherate R, Ashe JH (1993) Ionic flux contributions to neocortical slow waves and nucleus basalis-mediated activation: whole-cell recordings in vivo. J Neurosci 13:5312-5323.

Mukovski M, Chauvette S, Timofeev I, Volgushev M (2007) Detection of active and silent states in neocortical neurons from the field potential signal during slow-wave sleep. Cereb Cortex 17:400-414.

Nicholas AP, Pieribone V, Hökfelt T (1993) Distributions of mrnas for alpha-2 adrenergic receptor subtypes in rat brain: an in situ hybridization study. J Comp Neurol 328:575-594.

Nir Y, Staba RJ, Andrillon T, Vyazovskiy VV, Cirelli C, Fried I, Tononi G (2011) Regional slow waves and spindles in human sleep. Neuron 70:153-169.

Okun M, Naim A, Lampl I (2010) The subthreshold relation between cortical local field potential and neuronal firing unveiled by intracellular recordings in awake rats. J Neurosci 30:4440-4448.

Payne JD, Nadel L (2004) Sleep, dreams, and memory consolidation: the role of the stress hormone cortisol. Learn Mem 11:671-678.

Pirker S, Schwarzer C, Wieselthaler A, Sieghart W, Sperk G (2000) Gabaa receptors: immunocytochemical distribution of 13 subunits in the adult rat brain. Neuroscience 101:815-850.

Potez S, Larkum ME (2008) Effect of common anesthetics on dendritic properties in layer 5 neocortical pyramidal neurons. J Neurophysiol 99:1394-1407.

Poulet JF, Petersen CC (2008) Internal brain state regulates membrane potential synchrony in barrel cortex of behaving mice. Nature 454:881-885.

Pralong E, Magistretti PJ (1995) Noradrenaline increases k-conductance and reduces glutamatergic transmission in the mouse entorhinal cortex by activation of alpha 2-adrenoreceptors. Eur J Neurosci 7:2370-2378.

Reinoso-Suarez F (1961) Topographischer hirnatlas der katze, fur experimental-physiologische untersuchungen. Darmstadt: Merck.

Rudolph M, Pospischil M, Timofeev I, Destexhe A (2007) Inhibition determines membrane potential dynamics and controls action potential generation in awake and sleeping cat cortex. J Neurosci 27:5280-5290.

Rudolph U, Antkowiak B (2004) Molecular and neuronal substrates for general anesthetics. Nat Rev Neurosci 5:709-720.

Sanchez-Vives MV, Mattia M, Compte A, Perez-Zabalza M, Winograd M, Descalzo VF, Reig R (2010) Inhibitory modulation of cortical up states. J Neurophysiol 104:1314-1324.

Sharma AV, Wolansky T, Dickson CT (2010) A comparison of sleeplike slow oscillations in the hippocampus under ketamine and urethane anesthesia. J Neurophysiol 104:932-939.

Sonohata M, Furue H, Katafuchi T, Yasaka T, Doi A, Kumamoto E, Yoshimura M (2004) Actions of noradrenaline on substantia gelatinosa neurones in the rat spinal cord revealed by in vivo patch recording. J Physiol 555:515-526.

Steriade M, McCarley RW (2005) Brainstem control of wakefulness and sleep. New York: Plenum.

Steriade M, Nuñez A, Amzica F (1993a) Intracellular analysis of relations between the slow $(<1 \mathrm{~Hz})$ neocortical oscillations and other sleep rhythms of electroencephalogram. J Neurosci 13:3266-3283.

Steriade M, Nuñez A, Amzica F (1993b) A novel slow $(<1 \mathrm{~Hz})$ oscillation of 
neocortical neurons in vivo: depolarizing and hyperpolarizing components. J Neurosci 13:3252-3265.

Steriade M, Contreras D, Curró Dossi R, Nuñez A (1993c) The slow $(<1$ $\mathrm{Hz}$ ) oscillation in reticular thalamic and thalamo-cortical neurons: scenario of sleep rhythm generation in interacting thalamic and neocortical networks. J Neurosci 13:3284-3299.

Steriade M, Timofeev I, Grenier F (2001) Natural waking and sleep states: a view from inside neocortical neurons. J Neurophysiol 85:1969-1985.

Timofeev I, Steriade M (1996) Low-frequency rhythms in the thalamus of intact-cortex and decorticated cats. J Neurophysiol 76:4152-4168.

Timofeev I, Contreras D, Steriade M (1996) Synaptic responsiveness of cortical and thalamic neurones during various phases of slow sleep oscillation in cat. J Physiol 494:265-278.

Timofeev I, Grenier F, Steriade M (2001) Disfacilitation and active inhibition in the neocortex during the natural sleep-wake cycle: an intracellular study. Proc Natl Acad Sci U S A 98:1924-1929.

Tononi G, Koch C (2008) The neural correlates of consciousness. An update. Ann N Y Acad Sci 1124:239-261.

Volgushev M, Chauvette S, Mukovski M, Timofeev I (2006) Precise long- range synchronization of activity and silence in neocortical neurons during slow-wave sleep. J Neurosci 26:5665-5672.

Vyazovskiy VV, Olcese U, Lazimy YM, Faraguna U, Esser SK, Williams JC, Cirelli C, Tononi G (2009) Cortical firing and sleep homeostasis. Neuron 63:865-878.

Vyazovskiy VV, Olcese U, Hanlon EC, Nir Y, Cirelli C, Tononi G (2011) Local sleep in awake rats. Nature 472:443-447.

Wang M, Ramos BP, Paspalas CD, Shu Y, Simen A, Duque A, Vijayraghavan S, Brennan A, Dudley A, Nou E, Mazer JA, McCormick DA, Arnsten AF (2007) Alpha2a-adrenoceptors strengthen working memory networks by inhibiting camp-hon channel signaling in prefrontal cortex. Cell 129:397-410.

Wilson CJ, Kawaguchi Y (1996) The origins of two-state spontaneous membrane potential fluctuations of neostriatal spiny neurons. J Neurosci 16:2397-2410.

Wilson CJ, Chang HT, Kitai ST (1983) Disfacilitation and long-lasting inhibition of neostriatal neurons in the rat. Exp Brain Res 51:227-235.

Wolansky T, Clement EA, Peters SR, Palczak MA, Dickson CT (2006) Hippocampal slow oscillation: a novel EEG state and its coordination with ongoing neocortical activity. J Neurosci 26:6213-6229. 